\title{
Uso de levodopa em pacientes com ambliopia
}

\author{
Levodopa use in patients with amblyopia
}

\author{
Edson Procianoy (1), Flávio D. Fuchs ${ }^{(2)}$, Fernando Procianoy ${ }^{(3)}$, Letícia Procianoy ${ }^{(4)}$
}

\section{INTRODUÇ̃̃̃O}

Ambliopia é definida como uma diminuição da acuidade visual unilateral (o mais freqüente) ou bilateral, decorrente de deprivação visual e ou de interação binocular anormal. Não tem causa orgânica detectável ao exame físico do olho, ocorrendo no período de imaturidade do sistema visual. É reversível quando tratada em tempo e de modo apropriado.

Diagnostica-se ambliopia quando a diferença de visão entre os olhos é de duas ou mais linhas ou quando a visão é 20/30 ou pior na escala de Snellen.

Estrabismo e anisometropia são as causas mais freqüentes de ambliopia, ocorrendo em 2 a $5 \%$ da população ${ }^{1}$. Nos estrabismos, encontra-se ambliopia em $60 \%$ dos casos.

A visão se desenvolve nos seis primeiros anos de vida. A plasticidade sensorial é maior nos dois primeiros anos, isto é, até esta idade qualquer obstáculo ao desenvolvimento da visão causa diminuição rápida da acuidade visual, assim como o tratamento neste período promove pronta recuperação. Após os quatro anos de idade esta plasticidade é significativamente menor, resultando em ambliopia mais intensa e de mais difícil tratamento. Ching, Parks e Friendly ${ }^{2}$, demonstraram que a melhora da visão do olho amblíope se mantém quando a ambliopia é eliminada nos primeiros anos de vida e monitorizada com oclusão parcial, quando necessário, até os 9 anos de idade. Embora ainda se discuta a idade limite que é possível recuperar ambliopia, considera-se como irreversível a ambliopia não tratada em crianças acima de 8 anos de idade ${ }^{3}$. Nestes casos, a maioria não colabora com o tratamento de oclusão e a manutenção de uma melhora significativa é questionável.

O tratamento da ambliopia consiste, há mais de um século, na oclusão do olho de melhor visão para forçar o uso do olho amblíope. Assim sendo, dois fatores são importantes na

(1) Professor chefe do Setor de Estrabismo do Serviço de Oftalmologia da Universidade Federal do Rio Grande do Sul.

(2) Professor do Departamento de Medicina Interna da Faculdade de Medicina da Universidade Federal do Rio Grande do Sul.

3) Acadêmico da Faculdade de Medicina da UFRGS

(4) Acadêmica da Faculdade de Medicina da PUCRS

Os autores não têm interesse comercial ou financeiro no desenvolvimento ou marketing dos fármacos referidos no estudo.

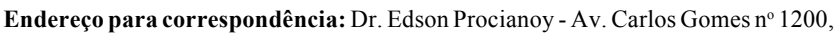
cj. 805. Porto Alegre (RS) CEP 91340-430. E-mail: edson@orion.ufrgs.br adesão ao tratamento: acuidade visual inicial e intensidade da ambliopia. Quanto melhor a visão inicial e menos tempo decorrer para se obter uma acuidade visual funcional, maior será a chance de sucesso do tratamento. Buscando obter maior efetividade do tratamento, bem como recuperar pacientes considerados irrecuperáveis, pesquisas tem sido feitas com tratamento farmacológico.

Nesta revisão discutem-se os estudos feitos com a principal abordagem farmacológica proposta com este objetivo, a associação de levodopa com carbidopa.

\section{LEVODOPA/CARBIDOPA NO TRATAMENTO DA AMBLIOPIA}

A levodopa é precursora da dopamina, um dos principais neurotransmissores do sistema extrapiramidal. Seu emprego tradicional é na doença de Parkinson, uma situação clínica classicamente atribuída à depleção de dopamina no estriado ${ }^{4}$. Pelo fato da dopamina não atravessar a barreira hematoencefálica, o tratamento que intenta aumentar suas concentrações no sistema nervoso central utiliza levodopa, que transportada através da barreira, transforma-se em dopamina. Como a levodopa também pode ser convertida em dopamina a nível periférico, ocasionando efeitos indesejáveis, administra-se carbidopa, simultaneamente, para inibir esta transformação.

A deprivação visual diminui a concentração de dopamina na retina de galinhas e macacos ${ }^{5}$. Estudos em animais amblíopes por deprivação sugeriram que os neurotransmissores estão envolvidos com a plasticidade da visão e podem restabelecer parcialmente a acuidade visual em gatos adultos ${ }^{5}$. No homem, foi detectada grande quantidade de dopamina nas células amácrinas e interplexiformes ${ }^{5}$. Nos pacientes com Parkinson, onde há deficiência generalizada de dopamina, observa-se alteração dos potenciais evocados e eletroretinograma, indicando a participação da dopamina na fisiologia da visão ${ }^{5}$.

O primeiro trabalho que estudou o efeito da levodopa em ambliopia no homem foi realizado por Gottlob e Stangler-Zuschrott ${ }^{5} \mathrm{em} 1990$. Foram estudados 9 pacientes adultos com ambliopia severa por estrabismo ou anisometropia em um estudo cruzado, duplo cego com grupo placebo controle. Observaram que após 90 minutos da ingestão de uma dose de $200 \mathrm{mg}$ de levodopa houve significativa melhora do teste de sensibilidade ao contraste 
bem como significativa diminuição do escotoma em relação ao período placebo.

Em 1991, Leguire e cols. ${ }^{6}$ apresentaram um estudo piloto para verificar a tolerância e a eficácia de levodopa/carbidopa. Participaram deste estudo 5 crianças ( 7 a 12 anos) com ambliopia (4 por anisometropia e uma por estrabismo) e dois adultos normais. Após a ingestão de uma dose de 100/25mg ou 400/100mg (levodopa/carbidopa respectivamente), correspondendo a dose de aproximadamente $4 \mathrm{mg} / \mathrm{kg}$ para as crianças e 5 e $8 \mathrm{mg} / \mathrm{kg}$ nos dois adultos, verificaram a ocorrência de efeitos adversos nas três primeiras horas e mediram a acuidade visual após 1 e 5 horas. Não foi observada nenhuma alteração de freqüência cardíaca, respiratória, pressão arterial e temperatura. Náusea e vômitos apareceram em 4 dos 7 casos nas primeiras duas horas. Após ingestão de alimentos com pouca proteína ( 2 horas após tomarem o medicamento) somente um paciente ainda teve náusea e vômito. A acuidade visual dos olhos amblíopes, medida pela escala de Snellen, mostrou melhora estatisticamente significativa, que foi máxima em 1 hora vindo a diminuir após 5 horas. A acuidade visual basal média, convertida em logaritmo, era 20/158 e passou para 20/83. O teste de sensibilidade ao contraste mostrou nos pacientes com ambliopia melhora em ambos os olhos, maior no amblíope do que no dominante e nos pacientes normais uma diminuição que não foi significativa. A análise dos potenciais evocados mostrou melhora em 3 dos 5 amblíopes e nenhuma alteração nos pacientes normais. A farmacocinética mostrou-se semelhante no adulto e na criança: máximo efeito entre 30 minutos e 1 hora após ingestão do medicamento, diminuindo em $50 \%$ após 2 a 4 horas. Estes resultados foram reapresentados em outra publicação, em $1992^{7}$.

Gottlob e cols. ${ }^{8}$, em 1992, publicaram um estudo cruzado, duplo-cego, com grupo placebo controle, para verificar o efeito de levodopa/carbidopa quando ingerida por uma semana. Empregaram a dose de $2 \mathrm{mg} / \mathrm{kg}$ de levodopa em três administrações diárias $(6 \mathrm{mg} / \mathrm{kg} / \mathrm{dia})$. Neste estudo participaram 20 pacientes (12 a 58 anos), que apresentavam ambliopia por anisometropia (4 casos), anisometropia e estrabismo ( 7 casos), estrabismo ( 8 casos), e estrabismo e nistagmo (1 caso). Os pacientes foram examinados após completar uma semana de uso de levodopa ou placebo e após um período de "wash-out" de uma semana, quando os pacientes cruzaram para o grupo oposto. Houve melhora da acuidade visual na semana de uso de levodopa em 70\% dos pacientes. Em 10 pacientes a melhora foi de menos de uma linha e em 4 foi de mais de uma linha (máximo foi uma linha e meia). Após esta segunda medida, a acuidade visual permaneceu significativamente melhor do que a basal nas três semanas seguintes (final do estudo). No grupo placebo, somente $25 \%$ melhorou a acuidade visual, não havendo melhora significativa da visão após uma semana de tratamento. $\mathrm{O}$ campo de visão melhorou entre o primeiro e o segundo exame no grupo que tomou levodopa e não no grupo placebo. $\mathrm{O}$ aumento da freqüência dos pacientes que melhoraram a acuidade visual neste estudo (70\%), quando comparado com o de dose única $(22 \%)$, pode ser atribuído a um maior efeito da dose prolongada e ou diferença entre os grupos. Comparando com o trabalho de Leguire e cols ${ }^{6,7}$, acreditam que estes obtiveram melhora mais acentuada da acuidade visual por empregarem dose maior e ou por empregarem em um grupo com idade menor de 12 anos. Concluem com a necessidade de se investigar o efeito de doses mais altas e de tratamento prolongado, bem como estudar um maior número de pacientes para saber porque alguns pacientes respondem mais ao tratamento.

Em 1993, Leguire e cols. ${ }^{9}$ apresentaram os resultados de uma investigação que visava determinar, em crianças, se doses menores de levodopa/carbidopa, quando associada com oclusão melhorava a acuidade visual com menor incidência de efeitos adversos (náusea e vômitos). Realizaram um estudo randomizado em paralelo, duplo cego controlado por placebo. Participaram 20 crianças (4 a 14 anos) sendo 6 com ambliopia por estrabismo, 9 por anisometropia e 5 por anisometropia e estrabismo. Tendo em vista que em estudo anterior ${ }^{6}$ levodopa/carbidopa mostrou maior efeito na melhora da acuidade visual do que na sensibilidade ao contraste e potenciais evocados, mediram neste trabalho somente a acuidade visual. Verificaram após 1 e 5 hs. o efeito das doses de $25 \mathrm{mg}(\mathrm{n}=6)$ e 50 $\mathrm{mg}(\mathrm{n}=8)$ de levodopa e compararam com um grupo que recebeu placebo $(n=6)$. Durante o estudo todos os pacientes ficavam com o olho dominante ocluído. Como o peso dos participantes variou de 15 até $60 \mathrm{~kg}$ a dose média dada ao grupo que recebeu $25 \mathrm{mg}$ de levodopa foi de $0,95 \mathrm{mg} / \mathrm{kg}$ (variabilidade não foi publicada) e a do grupo de $50 \mathrm{mg}$ foi $1,94 \mathrm{mg} / \mathrm{kg}(0,76$ até $3,014 \mathrm{mg} / \mathrm{kg})$. Analisaram o logaritmo da fração de Snellen e verificaram que a melhora da acuidade visual média no grupo placebo foi $0,063(\mathrm{SD}=0,073)$, não significativamente diferente de zero. No grupo que tomou 25/ $6,25 \mathrm{mg}$ e 50/12 $\mathrm{mg}$ de levodopa/carbidopa a melhora média foi respectivamente $0,143(\mathrm{SD}=0,079)$ e $0,125(\mathrm{SD}=0,124)$ e $\mathrm{P}<0,05$. Os pacientes com ambliopia por anisometropia tenderam a apresentar um melhor resultado do que os amblíopes por estrabismo. Também não houve correlação significativa do efeito com idade e dose baseada no peso $(\mathrm{mg} / \mathrm{kg})$. Não houve diferença de sintomas nos três grupos, ocorrendo náusea em $4 \%$ do grupo placebo, $3 \%$ no grupo que tomou $25 \mathrm{mg}$ e $1 \%$ no grupo que tomou $50 \mathrm{mg}$ de levodopa.

Leguire e cols. ${ }^{10}$, em 1993, dando continuidade a pesquisa de tolerabilidade e eficácia de levodopa/carbidopa, publicaram o primeiro trabalho em que crianças tomaram levodopa/carbidopa ao longo de 3 semanas. Tratou-se de estudo duplo cego, cruzado, com grupo placebo controle, com 10 crianças de 6 a 14 anos (5 ambliopias por estrabismo, 3 por anisometropia e 2 anisometropia com estrabismo) ao longo de 4 semanas. Devido a falta de conhecimento dos efeitos adversos de um tratamento prolongado em crianças, empregaram doses semanais crescentes por três semanas: 10/2,5 mg, 
20/5 mg e 30/7,5 mg de levodopa/carbidopa. Durante cada semana de maneira randomizada, um grupo recebia a dose descrita de $8 / 8$ hs. e outro placebo, e ficavam com o olho de melhor visão ocluído por $3 \mathrm{hs}$. no final do dia. Ao final de cada semana mediam a acuidade visual e questionavam a ocorrência de potenciais efeitos adversos. Não houve alteração significativa de peso, pressão arterial, temperatura, pulso e respiração entre os grupos. Não ocorreram episódios de náuseas e vômitos. Houve melhora da acuidade após concluída a terceira semana de medicamento em todos os grupos. O maior efeito foi no grupo que tomou $20 / 5 \mathrm{mg}$ (dose média $=0,48 /$ $0,12 \mathrm{mg} / \mathrm{kg}$ ) de levodopa/carbidopa, em segundo lugar no que tomou 10/2,5 mg (dose média $=0,24 / 0,06 \mathrm{mg} / \mathrm{kg}$ ) e o menor nos que tomaram 30/7,5 $\mathrm{mg}$ (dose média $=0,72 / 0,18 \mathrm{mg} / \mathrm{kg}$ ). Após uma semana sem medicamento (quarta semana), a acuidade visual do grupo que tomou levodopa/carbidopa comparada com a acuidade basal mostrou-se ainda significativa $(\mathrm{P}<0,03)$ o que não aconteceu com o grupo placebo $(\mathrm{P}>0,05)$.

Gottlob e cols. ${ }^{11}$, em 1995, apresentaram os resultados de um estudo duplo cego, em paralelo, com grupo placebo controle, envolvendo 29 pacientes ( 16 a 63 anos) com ambliopia por anisometropia e por estrabismo. O grupo tratado (14 pacientes) recebeu doses crescentes de levodopa/carbidopa nos primeiros 5 dias, iniciando com $6 \mathrm{mg} / \mathrm{kg} / \mathrm{dia}$ indo até 9 $\mathrm{mg} / \mathrm{kg} /$ dia. Esta dose foi dividida em três tomadas diárias e administrada por 3 semanas. Verificaram o campo de visão e a acuidade visual por quatro vezes (antes de iniciar a administração do fármaco, no final de três semanas, e um e dois meses após terminar o tratamento). Encontraram melhora significativa da acuidade visual e do campo de visão (diminuição do escotoma) no grupo tratado, entre os exames, e não no grupo placebo (15 pacientes). O aumento da dose de $6 \mathrm{mg} / \mathrm{kg} / \mathrm{dia}$ para $9 \mathrm{mg} / \mathrm{kg} / \mathrm{dia}$ bem como o seu emprego por maior tempo não intensificou a melhora da acuidade e três pacientes tiveram de interromper o uso por náuseas e vômitos. As melhoras da acuidade e campo visual persistiram por 2 meses após completar o tratamento com levodopa. Os autores concluíram com a necessidade de estender as observações, investigando, especialmente, a terapia de oclusão para aumentar o efeito da levodopa em pacientes adulto e a resposta de levodopa em crianças.

Em 1995, Leguire e cols. ${ }^{12}$ estudaram os efeitos da levodopa empregada por 7 semanas e após 6 semanas sem tratamento. Analisaram em 15 crianças o efeito da dose média de $0,55 \mathrm{mg} / \mathrm{kg}(0,45-0,60 \mathrm{mg} / \mathrm{kg})$ de levodopa com $25 \%$ de carbidopa, administradas em intervalos de 8 horas, associadas a oclusão de 3 horas por dia. A idade das crianças variou de 6 a 15 anos e a causa da ambliopia foi anisometropia em 7, estrabismo em 4, deprivação 2 e anisometropia com estrabismo em 2. A acuidade visual média basal no olho amblíope era $20 / 170(\log M A R=0,93)$. A acuidade melhorou sistematicamente nas primeiras 5 semanas de tratamento, estabilizandose nas duas semanas seguintes. A acuidade visual média, na quinta semana de tratamento, foi 20/103 $(\log M A R=0,71)$ e após a sétima semana foi 20/107 $(\log M A R=0,73)$. Após mais seis semanas sem tratamento a acuidade visual média permaneceu em 20/107 $(\log M A R=0,73)$. Esta melhora de acuidade visual foi estatisticamente significativa $(\mathrm{P}<0,01)$ quando comparada com a média da acuidade visual basal. As crianças com ambliopia por anisometropia e por estrabismo mostraram melhoras semelhantes. Ao término do tratamento, não houve correlação da melhora da acuidade visual com a idade dos pacientes, a acuidade basal e a adesão ao tratamento de oclusão. Foram descritos como efeitos adversos: náuseas $(\mathrm{N}=$ 9), cefaléia $(\mathrm{N}=9)$, mudança de comportamento $(\mathrm{N}=8)$, cansaço $(\mathrm{N}=5)$, boca seca $(\mathrm{N}=4)$ e vômitos $(\mathrm{N}=3)$. Os autores aventaram que estes sintomas possam ter decorrido de outros quadros clínicos concomitantes, como otites e resfriados. Peso, pressão arterial, temperatura, freqüência cardíaca e respiratória basais e hemograma não se alteraram. Concluíram com a necessidade de determinar a melhor dose, o efeito máximo passível de ser obtido em crianças mais velhas e em adultos, bem como realizar mais estudos sobre a influência dos neurotransmissores no sistema visual.

Leguire e cols (13), em 1998, estudaram o efeito da levodopa com e sem oclusão parcial. Estudaram 13 crianças de 7 a 12 anos (6 com ambliopia por estrabismo e anisometropia, 4 com ambliopia por anisometropia, 2 com ambliopia por estrabismo, e 1 com ambliopia por catarata congênita), que foram randomizadas à receber levodopa/carbidopa na dose média de $1,02 / 0,25 \mathrm{mg} / \mathrm{kg} 3$ vezes por dia, por 7 semanas. A acuidade visual do olho amblíope melhorou no grupo que fez oclusão 2,1 linhas $(\mathrm{P}<0,0001)$ e no grupo que não fez oclusão melhorou $0,8(P=0,037)$. A comparação entre os dois grupos mostrou que a melhora no grupo que fez oclusão é clinica e estatisticamente significativa $(P=0,002)$. Os autores concluíram que seus resultados sugerem que levodopa com oclusão parcial é mais efetiva do que somente levodopa em melhorar a acuidade visual de crianças mais velhas com ambliopia. A máxima melhora que pode ser obtida com levodopa foi colocada como uma questão a ser investigada.

Em 1999, Procianoy e cols. ${ }^{14}$ realizaram um estudo randomizado, duplo-cego, em paralelo e controlado por placebo, envolvendo 78 crianças amblíopes, exclusivamente por estrabismo, entre 7 a 17 anos de idade. As crianças foram designadas randomicamente a receber doses médias diárias de 0,51 , 1,05 ou $2,29 \mathrm{mg} / \mathrm{kg} / \mathrm{dia}$ por 1 semana, com oclusão do olho dominante por 3 horas/dia. A acuidade visual melhorou em logaritmo da fração de Snellen de 0,59 para 0,45 no grupo que usou levodopa/carbidopa e de 0,69 para 0,63 no grupo controle $(\mathrm{P}=, 023)$. Observou-se maior efeito no grupo que usou a menor dose de levodopa. A colaboração com a oclusão foi semelhante em todos os grupos e não ocorreram efeitos colaterais com as dosagens da droga administrada. Os autores concluíram que a dose média de $0,51 \mathrm{mg} / \mathrm{kg} / \mathrm{dia}$ é bem tolerada e produz melhora clínica e estatisticamente significativa a curto prazo na acuidade visual de crianças amblíopes. 
A possibilidade de alterar o período crítico de recuperação da visão, bem como facilitar o tratamento da ambliopia, tornam estes trabalhos como dos mais importantes em oftalmologia. Independentemente dos possíveis mecanismos pelos quais a levodopa atua, os resultados destes trabalhos mostraram que a levodopa melhora a acuidade visual em pacientes com ambliopia.

Determinar a duração ideal do tratamento, confirmar os efeitos de doses menores de levodopa, estudar o efeito da oclusão após o tratamento e identificar outros fatores que possam influenciar a resposta à medicação ainda são questões abertas sobre o efeito da levodopa em pacientes com ambliopia.

\section{REFERÊNCIAS BIBLIOGRÁFICAS}

1. Simons K, Reineke RD. Amblyopia screening and stereopsis. Transaction of the New Orleans Academy of Ophthal. Symposyum of Strabismus 1978;15-50.

2. Ching FC, Parks MM, Friendly DS. Practical management of amblyopia.J Pediatr Ophthalmol Strabismus 1986;23:12-6.

3. Paliaga GP. Controversies in functional amblyopia. Binocular Vision 1997; 12:155-66.
4. Fuchs FD, Wannmacher L. Antiparkinsonianos. Farmacologia Clínica, Editora Guanabara Koogan S.A. 1992;309-21.

5. Gottlob I, Strangler-Zuschratt E. Effect of levodopa on contrast sensitivity and scotomas in human amblyopia. Invest Ophthalmol Vis Sci 1990;31:776-80.

6. Leguire LE, Rogers GL, Bremer DL, Walson P, Wali N. Levodopa treatment for childhood amblyopia. Invest Ophthalmol Visc Sci 1991;32(suppl):820.

7. Leguire LE, Rogers GL, Bremer DL, Walson P, Hadjiconstantinou-Neff M. Levodopa and childhood amblyopia. J Pediatr Ophthalmol Strabismus 1992;29:290-8

8. Gottlob I, Charlier J, Reinecke RD. Visual acuities and scotomas after one week levodopa administration in human amblyopia. Invest Ophthalmol Vis Sci 1992;33:2722-8.

9. Leguire LE, Rogers GL, Bremer DL, Walson PD, McGregor ML. Levodopa/ carbidopa for childhood amblyopia. Invest Ophthalmol Vis Sci 1993;34:3090-5.

10. Leguire LE, Walson PD, Rogers GL, Bremer DI, McGregor ML. Longitudinal study of levodopa/carbidopa for childhood amblyopia. J Pediatr Ophthalmol Strabismus 1993;30:354-60.

11. Gottlob I, Wizov SS, Reinecke RD. Visual acuities and scotomas after 3 weeks' levodopa administration in adult amblyopia. Graefe's Arch Clin Exp Ophthalmol 1995;233:407-13.

12. Leguire LE, Walson PD, Roger GL, Bremer DL, McGregor ML. Levodopa/ carbidopa treatment for amblyopia in older children. J Pediatr Ophthalmol Strabismus 1995;32:143-9.

13. Leguire LE, Rogers GL, Walson PD, Bremer DL, McGregor ML. Occlusion and levodopa-carbidopa treatment for childhood amblyopia. J Pediatr Ophtalmol Strabismus, 1998;2:257-64.

14. Procianoy E., Fuchs FD, Procianoy L, Procianoy F. The effect of increasing doses of levodopa on children with strabismic amblyopia. J AAPOS, 1999;3(6):337-40.

\title{
I CONGRESSO PAN-AMERICANO DE TRAUMA OCULAR
}

\section{2 a 24 de Março de 2001}

\section{GRANDVILLE HOTEL Belo Horizonte-MG}

\author{
Promoção: Sociedade Pan-Americana de Trauma Ocular \\ Presidente: Dr. Christiano Barsante
}

\author{
Informações: Consult Comunicação e Marketing \\ Tel./Fax: (31) 3274-1550 \\ e-mail: comunica@consultcom.com.br \\ internet: www.consultcom.com.br
}

